\title{
Spontaneous Unilateral Achilles Tendon Rupture With Corticosteroid Use for Microscopic Polyangiitis
}

\author{
Jie Shen Fok ${ }^{a}$, Tuck Yean Yong ${ }^{\mathrm{a}, \mathrm{b}, \mathrm{c}}$, Jordan Yuanzhi Lia
}

\begin{abstract}
Achilles tendon rupture is increasingly reported in patients treated with corticosteroid and fluoroquinolones. We report about a patient who sustained a spontaneous non-traumatic Achilles tendon rupture while he was taking oral corticosteroid for the treatment of microscopic polyangiitis (MPA). This patient's tendon rupture was treated with a non-surgical approach because of his age, immunocompromised state and delay in presentation. The rupture healed gradually and his corticosteroid dose was weaned as the MPA improved.
\end{abstract}

Keywords: Achilles tendon; Corticosteroid; Microscopic polyangiitis; Rupture

\section{Introduction}

Spontaneous non-traumatic rupture of the Achilles tendon is rare but can be associated with long-term use of systemic corticosteroids [1], fluoroquinolones or both [2-5]. The most important risk factor for Achilles tendon rupture in young age group is related to sporting activities particularly recreational sports involving sudden acceleration and jumping [6]. However, spontaneous non-traumatic rupture of the Achilles tendon tends to occur in elderly patients. Rupture of the Achilles tendon is a serious condition that may lead to significant morbidity and often requires surgical treatment.

We report a case of spontaneous non-traumatic rupture

Manuscript accepted for publication February 8, 2013

${ }^{a}$ Departments of General Medicine, Flinders Medical Centre, Australia

${ }^{b}$ Faculty of Health Science, Flinders University, Adelaide, South

Australia, Australia

${ }^{\mathrm{c}}$ Corresponding author: Tuck Yean Yong, Department of General Medicine, Flinders Medical Centre, Flinders Drive, Bedford Park, South Australia 5042, Australia. Email: tuck.yong@health.sa.gov.au

doi: http://dx.doi.org/10.4021/jmc1270e of the Achilles tendon in a patient who is taking oral corticosteroids for the treatment of chronic kidney disease due to biopsy proven microscopic polyangiitis (MPA). We have also reviewed the literature on this topic.

\section{Case Report}

A 71-year-old man presented with a two-week history of fatigue, reduced appetite and urine output. He was found to be in acute kidney injury with serum creatinine of $590 \mu \mathrm{mol} / \mathrm{L}$ (reference range: $60-120 \mu \mathrm{mol} / \mathrm{L}$ ). His medical history was significant for hypertension and hyperlipidaemia for which he was on amlodipine $10 \mathrm{mg}$ daily and atorvastatin $20 \mathrm{mg}$ daily, respectively. Further investigations revealed urinary red cell casts and positive anti-neutrophil cytoplasmic antibodies (ANCA) on immunofluoresence with high titre of anti-myeloperoxidase (MPO) antibodies. Kidney biopsy demonstrated pauci-immune focal proliferative necrotising glomerulonephritis and crescent formation in keeping with MPA. He was treated with oral cyclophosphamide (150 mg daily) and pulse methyprednisolone ( $1 \mathrm{~g}$ daily for 3 days) followed by oral prednisolone $60 \mathrm{mg}$ daily, haemodialysis and 10 sessions of plasma exchange. His condition stabilised and renal function gradually improved. He became haemodialysis independent 14 days after admission. On discharged, he was taking oral cyclophosphamide $150 \mathrm{mg}$ daily and oral prednisolone $60 \mathrm{mg}$ daily, together with trimethoprim-sulfamethoxazole 160 - $800 \mathrm{mg}$ three times a week, calcitriol 0.25 mcg daily, and erythropoietin 6,000 units weekly. He was followed-up in the outpatient clinic. His renal function was stable with serum creatinine of $165 \mu \mathrm{mol} / \mathrm{L}$. The MPA was in remission 3 months later and azathioprine $75 \mathrm{mg}$ daily was commenced to replace cyclophosphamide as part of his maintenance immunosuppressive therapy. The prednisolone dose was gradually tapering.

He represented with 10-day history of extreme pain of the left ankle without trauma 8 months after initial presentation. The pain was exacerbated by walking and he was unable to place the entire left foot downward. He was taking prednisolone $10 \mathrm{mg}$ daily. On examination, there was absence of plantar flexion on performing calf compression test. There 
was a tender palpable gap in the tendon proximal to its insertion. The ultrasound revealed a markedly disorganised left Achilles' tendon with heterogeneous appearance throughout, suggesting full thickness retracted Achilles' tendon rupture. The tear commenced about $2 \mathrm{~cm}$ above the calcaneal insertion with markedly disorganised appearance extending over approximately $7 \mathrm{~cm}$ from that point to retracted Achilles' tendon ends. There was no evidence of active inflammatory arthritis. He was seen by an orthopaedic surgeon and adopted a non-surgical approach because of his age, immunocompromised status and delay in presentation. His prednisolone and azathioprine were discontinued 4 months later after tapering. On follow-up, the rupture Achilles' tendon has gradually healed and there was no evidence of relapse of MPA.

\section{Discussion}

Spontaneous non-traumatic rupture of the Achilles tendon is reported in association with rheumatoid arthritis [7], chronic kidney disease on dialysis [8], gout [9] and renal transplantation $[10,11]$. While a number of studies have highlighted the important role of quinolones in Achilles tendinopathy and complete tendon rupture [2-4], systemic corticosteroids as a contributing factor needs to be considered as well. The Achilles tendon rupture may occur on one or both sides.

Achilles' tendon rupture in a patient with MPA was previously described with the use of fluoroquinolones [5]. From our literature search, we believe this is the first case of Achilles' tendon rupture in a patient with MPA associated with the use of corticosteroid. The risk factors of Achilles' tendon rupture in our patient included being on corticosteroid, advanced renal failure, underlying connective tissue disease, male gender and age above 60 years [12].

Long-term oral corticosteroids have been implicated in causing Achilles tendon rupture. In one study, the annual incidence is estimated to be 1 in 600 patients on long-term oral corticosteroids for chronic airway obstruction [1]. In that study, no Achilles tendon rupture was reported among patients with chronic airways obstruction not receiving oral corticosteroids.

The mechanism of steroid-induced Achilles tendinopathy is not clearly understood. Suppressed repair mechanism of partially injured tendons may extend to complete rupture even after gentle locomotion [13]. Reduced tensile strength of the tendon due to collagen fibrils dysplasia as a result of steroid use has also been proposed [14]. Tendinopathy itself is an ongoing degenerative process [6]. It is possible that disruption of normal healing process by steroids may add to the already heavily diseased tendon, further contributing to its eventual rupture.

Achilles tendon rupture should be considered in any individual on long-term systemic corticosteroid treatment who presents with acute heel pain. The careful examination usu- ally reveals the presence of a bulging muscle belly in the proximal calf and a palpable gap in the Achilles tendon. Urgent referral for radiological investigation should be considered in this context to confirm the diagnosis.

Management options of Achilles' tendon rupture include both non-surgical and surgical approach. The former may involve static cast immobilisation and early functional rehabilitation whereas the latter often involves open or miniinvasive technique. The selection of approach should be individualised, depending upon the functional demand and medical condition of the patient [6], though recent studies suggest that surgical approach should probably be preferred [15].

High-dose systemic corticosteroid therapy, in association with other risk factors such as quinolone antibiotics, connective tissue disease, and severe renal impairment, predisposes to spontaneous Achilles tendon rupture. Clinicians should ensure that corticosteroids are prescribed appropriately and to advised patients of the risks associated with their use and to seek medical attention if suggestive symptoms develop.

\section{References}

1. Newnham DM, Douglas JG, Legge JS, Friend JA. Achilles tendon rupture: an underrated complication of corticosteroid treatment. Thorax. 1991;46(11):853-854.

2. van der Linden PD, Sturkenboom MC, Herings RM, Leufkens HM, Rowlands S, Stricker BH. Increased risk of achilles tendon rupture with quinolone antibacterial use, especially in elderly patients taking oral corticosteroids. Arch Intern Med. 2003;163(15):1801-1807.

3. Tsai WC, Yang YM. Fluoroquinolone-associated tendinopathy. Chang Gung Med J. 2011;34(5):461-467.

4. Lewis TG. A rare case of ciprofloxacin-induced bilateral rupture of the Achilles tendon. BMJ Case Rep. 2009;2009.

5. Sugimoto T, Kaneko H, Deji N, Koya D. Levofloxacin-induced Achilles tendon rupture in a patient with systemic microscopic polyangiitis. Mod Rheumatol. 2005;15(3):217-219.

6. Pingel J, Fredberg U, Qvortrup K, Larsen JO, Schjerling P, Heinemeier K, Kjaer M, et al. Local biochemical and morphological differences in human Achilles tendinopathy: a case control study. BMC Musculoskelet Disord. 2012;13:53.

7. Rask MR. Achilles tendon rupture owing to rheumatoid disease. Case report with a nine-year follow-up. JAMA. 1978;239(5):435-436.

8. Morein G, Goldschmidt Z, Pauker M, Seelenfreund M, Rosenfeld JB, Fried A. Spontaneous tendon ruptures in patients treated by chronic hemodialysis. Clin Orthop Relat Res. 1977;124:209-213. 
9. Mahoney PG, James PD, Howell CJ, Swannell AJ. Spontaneous rupture of the Achilles tendon in a patient with gout. Ann Rheum Dis. 1981;40(4):416-418.

10. Pirson Y, Ghysen J, Squifflet JP, Alexandre GP, van Ypersele de Strihou C. Multiple spontaneous ruptures of tendons in renal transplant recipient. Br Med J (Clin Res Ed). 1984;288(6422):1010.

11. Spencer JD. Spontaneous rupture of tendons in dialysis and renal transplant patients. Injury. 1988;19(2):86-88.

12. Sonohata M, Okamoto T, Uchihashi K, Motooka T, Tanaka H, Kitajima M, Mawatari M, et al. Subcutaneous Achilles tendon rupture in an eighty-year-old female with an absence of risk factors. Orthop Rev (Pavia). 2010;2(1):e11.

13. Haines JF. Bilateral rupture of the Achilles tendon in patients on steroid therapy. Ann Rheum Dis. 1983;42(6):652-654.

14. Kelly M, Dodds M, Huntley JS, Robinson CM. Bilateral concurrent rupture of the Achilles tendon in the absence of risk factors. Hosp Med. 2004;65(5):310-311.

15. Krapf D, Kaipel M, Majewski M. Structural and biomechanical characteristics after early mobilization in an Achilles tendon rupture model: operative versus nonoperative treatment. Orthopedics. 2012;35(9):e1383-1388. 